\title{
Hybrid III-V/IV Nanowires: High-Quality Ge Shell Epitaxy on GaAs Cores
}

Haotian Zeng, ${ }^{\dagger, \|}$ Xuezhe Yu, ${ }^{*}, \dagger, \|$ H. Aruni Fonseka, ${ }^{\star}$ James A. Gott, ${ }^{\star}$ Mingchu Tang, ${ }^{\dagger}$ Yunyan Zhang, ${ }^{\dagger}$ Giorgos Boras, ${ }^{\dagger}$ Jia Xu, ${ }^{\dagger}$ Ana M. Sanchez, ${ }^{\star}$ and Huiyun Liu ${ }^{\dagger}$

†Department of Electronic and Electrical Engineering, University College London, London WC1E 7JE, United Kingdom

Department of Physics, University of Warwick, Coventry CV4 7AL, United Kingdom

\begin{abstract}
The integration of optically-active III-V and electronic-suitable IV materials on the same nanowires could provide great potential for the combination of photonics and electronics in the nanoscale. In this paper, we demonstrate the growth of $\mathrm{GaAs} / \mathrm{Ge}$ core-shell nanowires on $\mathrm{Si}$ substrate by molecular beam epitaxy and investigate the radial and axial Ge epitaxy on GaAs nanowires in detail. High-quality core-shell nanowires with smooth side facets and dislocationfree, sharp interfaces are achieved. It is found that the low shell growth temperature leads to smoother side facets while higher shell growth temperatures lead to more relaxed structures with significantly faceted sidewalls. The possibility of forming III-V/IV heterostructure nanowire with Ge section development in the axial direction of GaAs nanowire using Ga droplet is also revealed. These nanowires provide an ideal platform for nanoscale III-V/IV combination which is promising for highly integrated photonic and electronic hybrid devices on a single chip.
\end{abstract}

Keywords: Nanowires, III-V/Group IV, hybrid heterostructure, MBE, single-crystalline

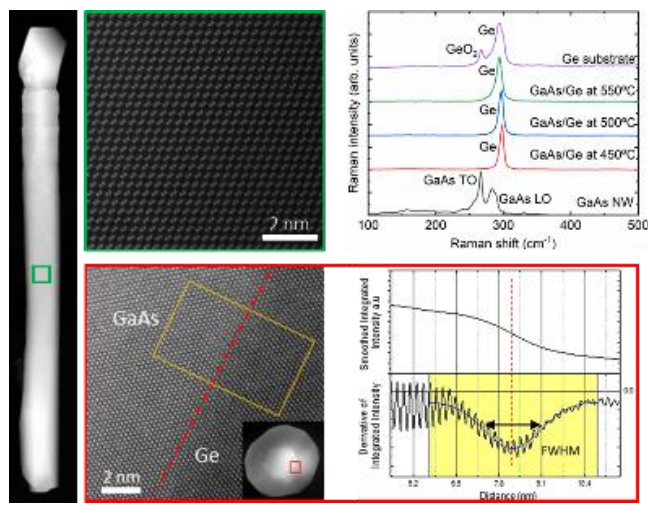

TOC 
Nanowires (NWs) have been demonstrated as promising building blocks for the nextgeneration electronics and photonics devices. ${ }^{1,2}$ The tremendous progress and continued development of nanowire structures in the last ten years are doubtlessly contributing towards the integrated circuit size reduction. ${ }^{1-4}$ In particular, semiconductor III-V and Group-IV nanowires have attracted great interest and attention. III-V nanowires, with direct bandgap and high absorption/emission characteristics, have shown encouraging results in photonic devices. They have been successfully demonstrated in solar cells, ${ }^{5,6}$ photodetectors, ${ }^{7,8}$ light-emitting diode and lasers. ${ }^{9-11}$ Meanwhile, Group-IV NWs are extremely suitable for nanoelectronics devices such as field-effect transistors. ${ }^{12-14}$ Their excellent thermal stability, inexpensive cost, and high mobility are ideal properties for success in modern electronics.

A key advantage of nanowires is the ability to combine materials in both axial and radial directions to produce new device architectures that would have been impossible in the planar architecture. Combining III-V and Group-IV materials on the same nanowire would provide a platform for innovative multifunctional optoelectronic and electronic devices that harness the advantages of both types of materials. Recently, research activities on hybrid III-V/IV NWs fast emerged with studies focusing on $\mathrm{Si} / \mathrm{InGaN}{ }^{15}$ and $\mathrm{GaAs} / \mathrm{Si}^{16}$ core-shell structures. Besides, the hybrid structures of $\mathrm{Si} / \mathrm{GaAs}^{17}$ and $\mathrm{Si} / \mathrm{GaP}^{18,19}$ in both axial and radial growth have also been demonstrated.

In heterostructures, a large lattice mismatch between materials can cause notable strain which could lead to the formation of dislocations at the heterostructure interface. In this case, latticematched materials are preferred for hybrid III-V/IV NW structures. When considering Si and Ge for the Group-IV materials, there are two preferential, lattice-matched materials available for the III-V binary candidate, namely GaP and GaAs, respectively. The lattice mismatches between the materials forming the $\mathrm{GaP}(5.451 \AA) / \mathrm{Si}(5.431 \AA)$ and $\mathrm{GaAs}(5.653 \AA) / \mathrm{Ge}(5.658$ $\AA$ ) hybrid structures are $-0.3 \%$ and $0.08 \%$, respectively. ${ }^{20}$ However, common zinc blende (ZB) $\mathrm{GaP}$ and Si both have an indirect bandgap, making the combination of $\mathrm{GaP} / \mathrm{Si}$ unusable in photonic device applications without resorting to direct bandgap wurtzite (WZ) $\mathrm{GaP}$ or using a GaAs insertion for the active region of the photonic device. ${ }^{18,19}$ Besides, the hexagonal Si grown on wurtzite GaP NWs suffers from low ductility causing "cracking defects" at high-temperature growth as well as other defects within Si shell. ${ }^{21}$ Instead, the alternative combination of $\mathrm{Ge}$ and GaAs, with only $0.08 \%$ lattice mismatch, could circumvent the problems in $\mathrm{GaP} / \mathrm{Si}$ combination and be more applicable in aforementioned photonic and electronic integrated applications.

In this work, we demonstrate the growth of GaAs/Ge core-shell NWs on Si substrates by molecular beam epitaxy. Sharp interface, smooth shell surface, and defect-free NW body are revealed by transmission electron microscope (TEM) inspection on radial growth. A Ge segment at the tip is also observed which confirms the axial growth of $\mathrm{Ge}$, catalyzed by $\mathrm{Ga}$ droplet. X-ray diffraction (XRD) measurements show that there are inappreciable mutual strain effects between Ge shell and GaAs NW. In addition, the Raman shift of Ge peaks from different growth temperature shows that strain is likely to be relaxed by facet formation at the higher growth temperatures.

Self-catalyzed GaAs NW cores were grown directly on p-type Si (111) substrates by the vapor-liquid-solid method using solid source III-V molecular beam epitaxy (MBE). A Ga beam pressure corresponding to a growth rate of 0.6 monolayers per second was used with a V/III flux ratio of 17. Under these conditions, the GaAs NW core growth was carried out at the 
growth temperature of $610{ }^{\circ} \mathrm{C}$ for 40 minutes. The growth was terminated by stopping both III and $\mathrm{V}$ solid sources simultaneously. The Ga catalyst droplet was preserved by doing this. After cooling down, the samples were transferred to the solid-source Group-IV MBE for Ge growth. By using the ultra-high-vacuum transfer chamber connecting the two MBE systems, the contamination was kept to a minimum without exposing GaAs NW samples to air. Four GaAs NWs samples were prepared with identical growth conditions mentioned; out of which, three GaAs NWs samples were continued with Ge shell growth at different temperatures $\left(450{ }^{\circ} \mathrm{C}\right.$, $500{ }^{\circ} \mathrm{C}$, and $550{ }^{\circ} \mathrm{C}$ ), using a Ge flux rate of $1.57 \times 10^{-7}$ Torr for 1 hour which corresponds to $0.282 \mathrm{ML} / \mathrm{s}$. The NW morphology was first inspected using a Zeiss XB 1540 Scanning Electron Microscope (SEM). Transmission electron microscopy (TEM) imaging and analysis were performed on NWs transferred to holey carbon grids using JEOL 2100 and doubly corrected ARM200F microscopes, both operating at $200 \mathrm{kV}$. Raman spectra were obtained in a Renishaw InVia Raman microscope, with a wavelength of $532 \mathrm{~nm}$ and an output power of $46 \mathrm{nW}$. Note that 10 accumulations were carried out for each measurement. Additional data to determine the composition and lattice parameters of nanowires were obtained using a Jordan Valley's D1 Evolution X-ray diffraction (XRD) instrument operating at $50 \mathrm{kV}$ power and $35 \mathrm{~mA}$ current.

Side-view SEM images of GaAs NWs grown on $\mathrm{Si}$ (111) substrates are shown in Figure 1a. The NWs showed optimized morphology with a uniform diameter along the NW. The droplets were intentionally retained to investigate the axial growth during Ge shell growth. A slight inversely tapered structure was observed in the subsequent GaAs/Ge core-shell NWs (Figure 1b-d). The uniform diameter of GaAs NWs (Figure 1a) indicates that the shape of GaAs NW is not responsible for the tapering of GaAs/Ge core-shell heterostructures. The shadowing effect could be one of the possible reasons for the tapering of GaAs/Ge NWs. This could be eliminated by using patterned substrates with more space between the holes. A reduction in Ga droplet size or even droplet disappearance from the NW tip was observed during the Ge shell growth at different temperatures. In addition, the faceting of the Ge shell noticeably increased with higher shell growth temperature according to the SEM images in Figure 1b-d. In contrast to the GaAs core, which has a typical hexagonal cross-section with $\{110\}$ side facets, the core-shell nanowires with shells grown at $450{ }^{\circ} \mathrm{C}$ have a combination of $\{110\}$ and $\{112\}$ side facets with varying relative proportions forming a dodecagonal cross-section shape (inset of Figure 1b). This observation is consistent with that generally observed for Ge and Si nanowires, where $\{110\}$ facets are partially transformed into $\{112\}$ facets due to lower surface energy of the $\{112\}$ facets. ${ }^{22} \mathrm{Ge}$ shells grown at $500{ }^{\circ} \mathrm{C}$ tend to form microfacets that are non-parallel to the growth direction and the trend continues in shells grown at the highest temperature of $550{ }^{\circ} \mathrm{C}$. This observation is again consistent with the behavior of Group-IV NWs that form $\{111\}$ microfacets to further reduce the total surface energy. ${ }^{22-24}$ In this case, the higher growth temperature has provided the required kinetic energy for the Ge atoms to overcome the kinetic energy barrier for surface migration in order to achieve lower surface energies. ${ }^{25}$

A statistical analysis is carried out on fifty GaAs/Ge NWs in terms of length, core radius and shell thickness and summarized in Figure S1 (supporting information). The GaAs core radius and GaAs/Ge NW radius can be readily obtained from SEM images, respectively, but with a higher magnification. As we have used the same growth condition for GaAs reference NWs growth (Fig. 1a) and GaAs core NWs growth for GaAs/Ge core-shell NWs growth (Fig. 1b-d), the radius of GaAs core can be regarded as the same for all samples. The average GaAs core radius was $52 \pm 4 \mathrm{~nm}$. The Ge shell thickness was measured by calculating the difference between the radius of GaAs/Ge NWs and that of GaAs NWs. The measured Ge shell thickness was $52 \pm 4.5 \mathrm{~nm}, 81 \pm 6.5 \mathrm{~nm}$, and $86 \pm 7 \mathrm{~nm}$ for shell growth temperatures of $450{ }^{\circ} \mathrm{C}, 500{ }^{\circ} \mathrm{C}$, 
and $550{ }^{\circ} \mathrm{C}$, respectively (Figure S1a, supporting information). Likewise, the length of the GaAs/Ge NWs increased by $0.24 \pm 0.01 \mu \mathrm{m}, 0.41 \pm 0.02 \mu \mathrm{m}$, and $0.62 \pm 0.03 \mu \mathrm{m}$ compared to the GaAs core NWs of $2.37 \pm 0.12 \mu \mathrm{m}$ for shell growth temperatures of $450{ }^{\circ} \mathrm{C}, 500{ }^{\circ} \mathrm{C}$, and $550{ }^{\circ} \mathrm{C}$, respectively (Figure S1b, supporting information). Higher temperatures provide higher kinetic energy and mobility for Ge atoms. Thus, more active $\mathrm{Ge}$ atoms impinged on the substrate surface can migrate to the nanowire and adhere to the surface of NW, resulting in an increase of thickness. Additionally, there is a minor difference in lengths between the GaAs and GaAs/Ge NWs. Since Ga droplet remained after GaAs NW growth, a Ge segment is expected at the NW tip due to additional Ge axial growth during the Ge shell radial growth. This has been confirmed in this work which will be elaborated later in this article. This axial growth has caused core-shell nanowires to be slightly longer than the GaAs cores.

TEM specimen and compositional analysis were carried out to evaluate the core-shell interface quality after sectioning the NW perpendicular to its growth direction using an ultramicrotome. Figure 2 a corresponds to a section of a NW with $450{ }^{\circ} \mathrm{C}$ Ge shell growth temperature. Uniform distributions of Ga (Pink) and As (cyan) in the core and Ge (purple) in the shell are shown in the same figure. Notice that the side facets are different in the core and in the shell as previously discussed. High magnification Annular Dark Field Scanning Transmission Electron Microscopy (ADF-STEM) image in Figure 2b shows the GaAs/Ge radial interface of a NW with shell grown at $450^{\circ} \mathrm{C}$. No indication of any extra planes, i.e. no misfit dislocations are revealed in the core-shell interface for this or either of the other two sample with 500 and $550{ }^{\circ} \mathrm{C}$ shell growth temperatures (See Figure S2 in supporting information). Figure $2 \mathrm{c}$ shows the integrated intensity profile taken across the interface of the NW cross-section from the yellow square marked in Figure $2 \mathrm{~b}$. The average thickness of the interface, calculated by the full width at half maximum (FWHM) of the derivative of the intensity line profile is around $2.7 \mathrm{~nm}$ for this sample (Figure 2d). Similar average values are obtained for the other two samples by measuring at least 3 interfaces per sample. This indicates that a reasonably sharp and defect-free interface has been achieved between the GaAs core and the Ge shell, and the variation of the interface quality with the shell growth temperature is minimal in the temperature range discussed.

Additional side and cross-sectional TEM specimen (i.e. perpendicular and parallel to the growth direction) were used to analyze the Ge heteroepitaxy on GaAs NWs. Low magnification TEM images showed a low density of planar defects that are stemming from the GaAs core in the three GaAs/Ge core-shell samples (Figure 3a-c). Here again, the images confirmed the increase in NW faceting with the Ge growth temperature. Sidewalls parallel to the growth direction were observed in the sample with shell grown at $450{ }^{\circ} \mathrm{C}$ (Figure $3 \mathrm{a}$ ), whereas a jagged morphology was revealed in the higher shell growth temperature samples (Figure $3 \mathrm{~b}$ and c). As previously discussed, higher growth temperatures induce faceting in Ge shell. A more detailed observation of Figure $3 \mathrm{~b}$ and $\mathrm{c}$, unfold that there is a loose relationship between the Ge shell and twin defects in the core NW. As it can be observed in the TEM images, there are faceting with and without a link to the twin defects (indicated with red and green arrow respectively). This suggests that some twin defects may have acted as preferred nucleation sites for the Ge shell while reduction of total surface energy in the Group-IV shell remains the main cause of faceting at high temperatures.

The ADF-STEM images revealed a high density of twin and stacking faults mainly in the region beneath the NW tip (Figure 3d) of all three samples. The rest of the Ge/GaAs NW body had a ZB structure in Figure 3e, with occasional twin defects. The elemental distribution in the 
NWs was examined using energy-dispersive X-ray (EDX) spectroscopy. EDX side view analysis on a middle region of a GaAs/Ge NW grown at $450{ }^{\circ} \mathrm{C}$ can be found in Figure $3 \mathrm{f}$. Ga (pink), As (cyan), and Ge (purple) maps clearly demonstrate the core-shell structure. EDX linescan (Figure 3g) recorded along the line marked with a white arrow in Figure $3 f$ further confirms the core-shell structure in these samples.

The tip area of the GaAs/Ge NWs has been investigated in detail by electron microscopy to determine the effect of the Ga droplet preservation during the Ge shell growth. The resulting GaAs/Ge NWs can roughly be divided into two main groups: (i) ones with no droplet left (Figure 4a) and (ii) those with Ga droplets of reduced size (Figure 4b) in comparison to the original GaAs NWs. High-resolution STEM images revealed the presence of a grain boundary originating from the bottom part of the Ge tip (red square area marked in Figure 4a) in some of the nanowires. In the inset of Figure 4a, Selective-area electron diffraction (SAED) image of the core-shell part right beneath the Ge tip shows two mirrored hexagons electron diffraction pattern, indicating the presence of twin defects. It has been frequently reported that NW core acts like a crystal-structure template, which leads the coated shell to follow the core's crystal structure in most situations. ${ }^{26-28}$ The twin defects can be attributed to the drastic volume change in Ga droplets at the end of the GaAs core growth, ${ }^{29}$ and Ge shell inherits the twin defects from the core. Therefore, to achieve a pure ZB Ge shell, a pure ZB GaAs NW core is essential. The grain boundary (Figure $4 \mathrm{c}$ ) generally lays in a plane at approximately $70^{\circ}$ respect to the $(\overline{1} \overline{1} \overline{1})$ NW growth direction, i.e. in the $(\overline{1} 1 \overline{1})$ planes. The Ge tip area apart from the grain boundary shows a pure ZB structure, as can be extracted from the high-resolution STEM image in Figure $4 \mathrm{~d}$. To understand the origin of the grain boundary feature observed in the Ge tip, side view EDX mapping was performed on a NW with shell growth temperature of $450{ }^{\circ} \mathrm{C}$, in the area corresponding to the transition section between Ge tip and core NW (Figure 4e-g). It can be observed that the tip is mainly Ge in composition with the GaAs/Ge core-shell structure underneath. Closer inspection of the elemental maps demonstrates the formation of a small GaAs island-like segment in the area close to the Ge tip (marked by the dash-line in Figure $4 \mathrm{f}$ and g) surrounded by Ge. It is seen that a $\{111\}$ step facet is formed where the GaAs and Ge come together. By comparing GaAs island-like segment with the outline of grain and twin boundary in the STEM image (marked by the dash-line in Figure 4h), it can be concluded that the grain boundary originates in the vicinity of the GaAs-Ge intersection (also see Figure S3 in supporting information).

XRD measurements corresponding to the GaAs/Ge NWs grown at $450{ }^{\circ} \mathrm{C}$ (Figure 5a), showed a shift in the peak location respect to the bare GaAs NWs, indicating the contribution of Ge to the average lattice parameter of the core-shell nanowires. Studies using first-principal approach have suggested that lattice constants of GaAs and Ge NWs within certain diameters can be considered to be the same as the strain-free bulk materials. ${ }^{30,31}$ Hence here, the strain in the core-shell structure is evaluated by the difference between the lattice constants calculated based on the GaAs/Ge XRD data and the strain-free lattice constants of bulk materials. By fitting peaks to the GaAs/Ge core-shell XRD data (Figure $5 \mathrm{~b}$ ), peaks corresponding to GaAs $\left(X_{1}\right)$ and $\mathrm{Ge}\left(X_{2}\right)$ with lattice constants of $5.6538 \AA$ and $5.6574 \AA$ are obtained respectively. This reveals that GaAs core is $0.01468 \%$ under tensile strain and Ge shell $0.00990 \%$ under compressive strain, which are negligible. These slight changes in lattice parameters, i.e. strain, has not been high enough to generate dislocations as previously demonstrated. Additionally, Raman spectroscopy (Figure 5c) showed a slight shift in the Ge peak of the three GaAs/Ge NW samples when compared to that of Ge substrate. The Raman Ge peaks are located at 297.8 $\mathrm{cm}^{-1}, 297 \mathrm{~cm}^{-1}$, and $293 \mathrm{~cm}^{-1}$, for the $450{ }^{\circ} \mathrm{C}, 500^{\circ} \mathrm{C}$, and $550^{\circ} \mathrm{C}$ shell growth temperatures, 
respectively, confirming that $\mathrm{Ge}$ is close to the full relaxation $\left(292 \mathrm{~cm}^{-1}\right)$. Ge shell growth at highest growth temperature presented the closest value to that of Ge substrate (293 vs 292 $\mathrm{cm}^{-1}$ ). Since no dislocations were observed in all three core-shell structures, the accumulated strain has been probably released by the facets formed at the shell surface. This agrees with the higher faceting degree observed in the NW with the Ge shell grown at the highest growth temperature. Besides, the GaAs peak from the reference (bare GaAs core) NWs is at $267 \mathrm{~cm}^{-1}$ which is in accord with the measurement by S. V. Karpov, ${ }^{32}$ while the GaAs peak from GaAs/Ge NWs cannot be seen from Raman spectroscopy. It has been reported that GaAs peak from Raman spectroscopy will be negligible compared to that of Ge film with a certain thickness. ${ }^{33-35}$ Similarly, it is also expected that with a full coverage of Ge shell, the peak of GaAs core will not be seen. In the core-shell NW (crystalline solids), the accumulated strain would lead to dislocations formation at core-shell interface during strain relaxation. The dislocations act as non-radiative recombination centers, degrading the optical and electrical performance. In GaAs/Ge core shell nanowires, the strain on both core or shell is negligible in theory and experimental results, which is also accords with the observation of zero dislocation at the interface shown in Figure 2.

In Figure 5d, the photoluminescent (PL) spectrum of bare GaAs NW sample and three GaAs/Ge NW samples are shown. All of the GaAs/Ge NW samples show a GaAs peaks around $870 \mathrm{~nm}$ as the bare GaAs NW in spite of the weaker intensity. The intensity of GaAs peak from bare GaAs NW sample is about 2 times larger than that of all GaAs/Ge NW samples. This is probably due to (i) Ge absorbing light without emitting light and leading less light to the GaAs core and (ii) more significantly, the photon-generated carriers diffuse to the Ge which has a narrower band gap compared to GaAs and recombining non-radiatively there. It is expected that the adoption of another lattice-matched material, like AlGaAs or AlAs between GaAs and $\mathrm{Ge}$, would alleviate the loss of photon-generated carriers by forming a barrier to confine the carriers effectively in the GaAs core. Decreasing the Ge shell thickness could also help to limit the light absorption by Ge shell. Noticeably, a broad peak around $1 \mu \mathrm{m}$ shown in GaAs NW reference sample. This wide peak, which is probably due to surface recombination, disappears after Ge coating. Thus, Ge shell provides another advantage as a surface passivation layer.

In conclusion, high-quality GaAs/Ge core-shell NWs featuring the smooth side facets, a sharp interface between core and shell with no misfit dislocations and negligible strain have been demonstrated by MBE. A lower growth temperature at $450^{\circ} \mathrm{C}$ yields better morphology and quality of Ge shell. With a higher temperature at $550{ }^{\circ} \mathrm{C}$, the smoothness of the side surface is degraded, though no other form of shell quality degradation such as dislocations is observed. It can be expected that the realization of nanophotonics and nanoelectronics in a unified platform would benefit from this combination of III-V and Group-IV materials radially on the same NW.

\section{ASSCIATED CONTENT}

\section{Supporting information}

Statistical morphological analysis on NWs in terms of radius, length and Ge shell thickness, additional information on $\mathrm{GaAs} / \mathrm{Ge}$ core-shell interface quality, and description of grain boundary formation mechanics

\section{AUTHOR INFORMATION:}




\section{Corresponding author}

*Email: xuezhe.yu@ucl.ac.uk

\section{Author Contribution}

" H.T. Zeng and X.Z. Yu contributed equally to this work.

Notes

The authors declare no competing financial interest.

\section{Acknowledgment:}

The authors acknowledge the support of Leverhulme Trust, the UK Engineering and Physical Sciences Research Council - EPSRC (grant nos. EP/P000916/1 and EP/ P000886/1), and EPSRC National Epitaxy Facility. 


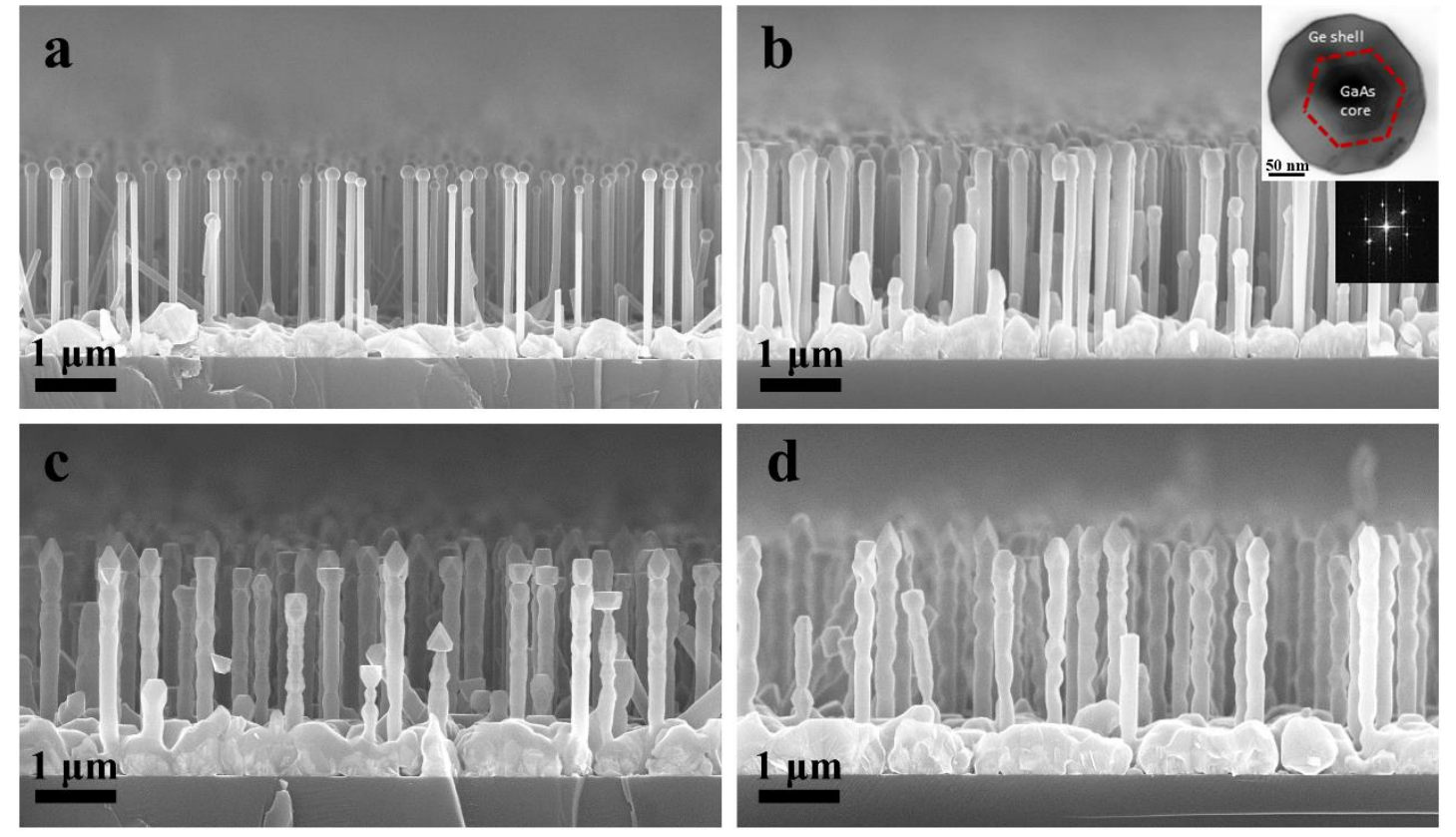

Figure 1. GaAs and GaAs/Ge NWs. (a) SEM image of GaAs NWs with Ga droplet, which are used to grow Ge shells. (b-d) SEM images of GaAs/Ge core-shell NWs with shells grown at $450{ }^{\circ} \mathrm{C}, 500{ }^{\circ} \mathrm{C}$, and $550{ }^{\circ} \mathrm{C}$, respectively. Inset of Figure $1 \mathrm{~b}$ shows a bright-field STEM image and the corresponding FFT from a cross-section from the same sample. 

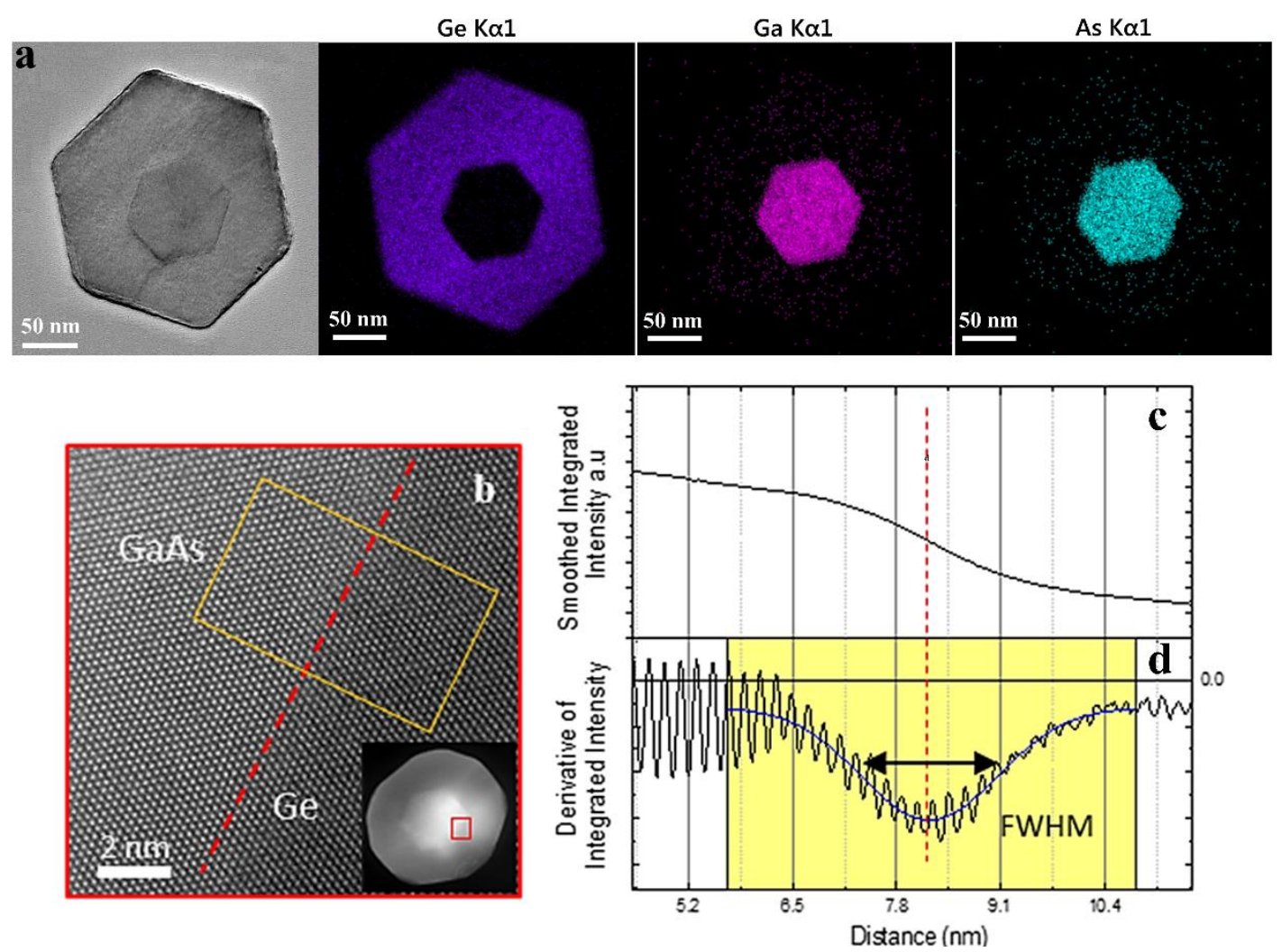

Figure 2. Radial Ge growth investigation using cross-sections (a) EDX mapping of a lateral cross-section of a GaAs/Ge NW with shell grown at $450^{\circ} \mathrm{C}$. (b) ADF-STEM image of GaAs/Ge radial interface of a NW with $450^{\circ} \mathrm{C}$ shell growth temperature. The red square in the inset shows the region magnified in the main figure. The yellow square indicates the region used to generate the integrated intensity profile in (c). (c) The smoothed integrated intensity profile of the GaAs/Ge interface marked by the yellow square in (b). (d) The derivative of the smoothed integrated intensity. The blue curve indicates the Gaussian fit used to obtain the FWHM value that has been used to compare the thickness of the interface. All cross-section TEM images are taken from the middle of the NW. 


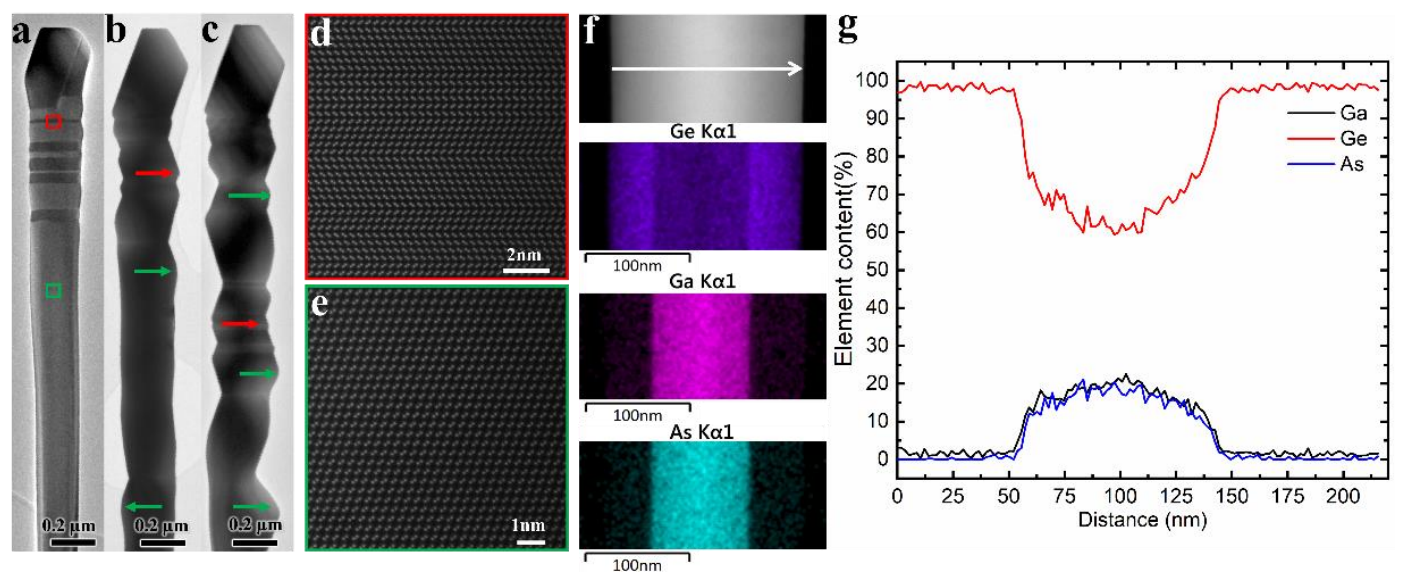

Figure 3. Investigation of Ge shell growth using the side view of GaAs/Ge NWs (a-c) Low magnification TEM images of single NWs from samples with shells grown at $450^{\circ} \mathrm{C}, 500^{\circ} \mathrm{C}$, and $550^{\circ} \mathrm{C}$. The red arrows point to the faceting associated with twin defects, while the green arrows point to the faceting that is not associated with twin defects. (d) ADF-STEM image of the red square area in (a), showing the twin defects below the Ge tip. (e) ADF-STEM image of the Ge shell marked by the green square in (a), showing the defect-reduced NW body with ZB structure. (f) EDX maps of a GaAs/Ge NW with shell growth temperature of $450^{\circ} \mathrm{C}$ : $\mathrm{Ga}$ (pink), As (cyan) and Ge (purple). (g) EDX line-scan element percentage of $\mathrm{Ga}, \mathrm{Ge}$ and As in the scan direction marked in (f). 


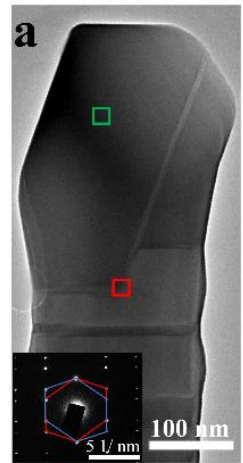

Ge K $\alpha 1$

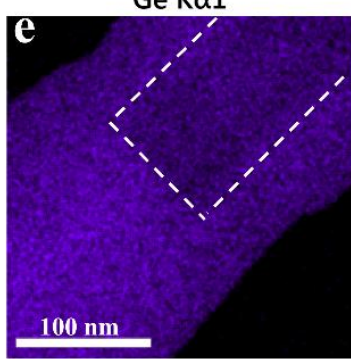

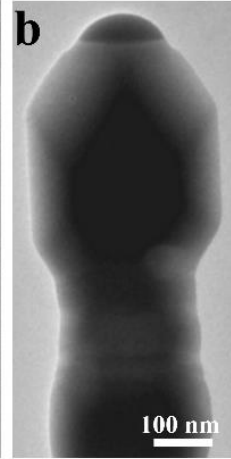

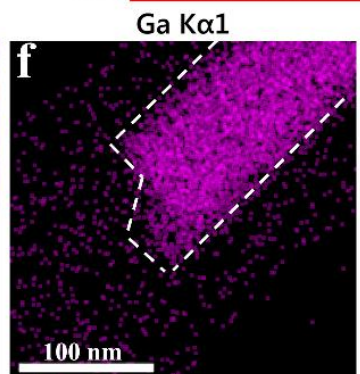

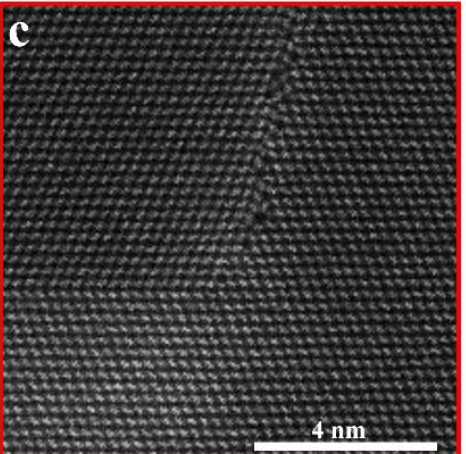

As $K \alpha 1$

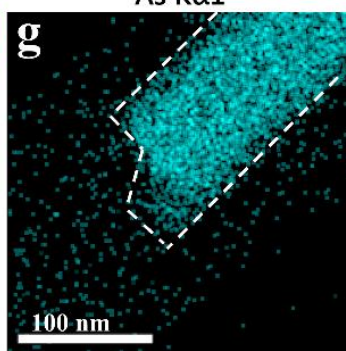

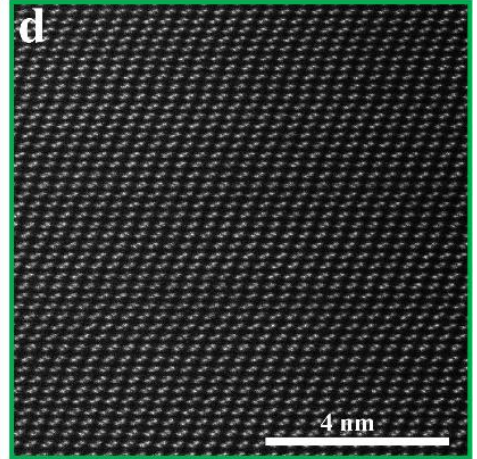

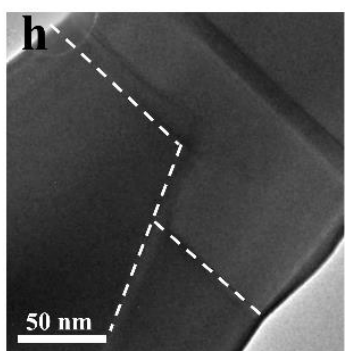

Figure 4. Investigation of Ge segment and the grain boundary within GaAs/Ge NW tip (a) TEM images of the NW tip region of the sample with $450{ }^{\circ} \mathrm{C}$ Ge shell growth temperature and no remaining Ga droplet. The inset is the SAED of the core-shell part right beneath the Ge tip. (b) TEM image of a NW tip region from the sample with $500{ }^{\circ} \mathrm{C}$ Ge shell growth temperature and with Ga droplet remaining. (c) ADF-STEM image of the grain boundary in (a). (d) ADFSTEM image of Ge tip area without grain boundary showing ZB structure. (e) Ge EDX map of NW tip in (a) showing a darker area marked by dash-line referring to the core. (f, g) Ga and As EDX maps of NW tip in (a), respectively, showing a similar outline of the GaAs core but with a small step on the end of the core marked by the dash-line. (h) A higher magnification TEM image of the grain boundary region, where the twin boundary and the grain boundary are marked by dash-line. 

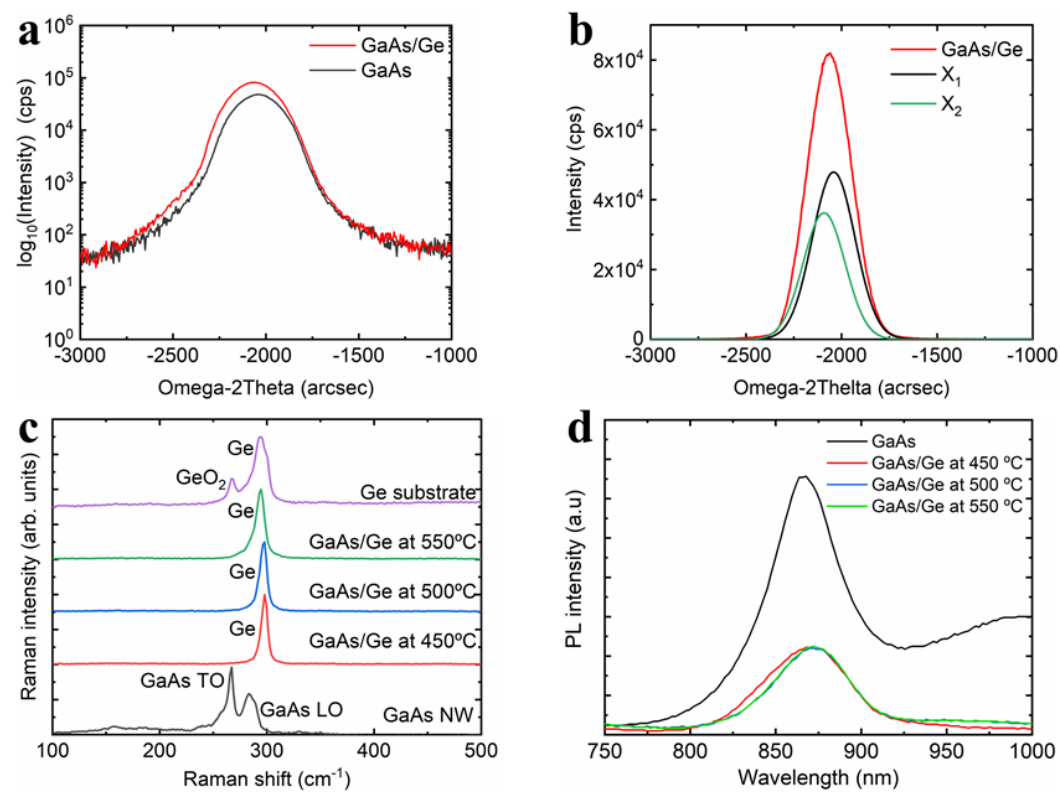

Figure 5. Strain analysis and photoluminescence (PL) of the GaAs NWs and GaAs/Ge NWs (a) XRD measurements for GaAs/Ge NWs with $450{ }^{\circ} \mathrm{C}$ Ge shell growth temperature and GaAs NWs on Si (111) in a common logarithm scale and (b) Peaks fitted to GaAs/Ge NWs with $450^{\circ} \mathrm{C} \mathrm{Ge}$ shell growth temperature in the linear scale showing the two peaks of GaAs $\left(X_{1}\right)$ and Ge $\left(X_{2}\right)$. (c) Raman spectroscopy of all samples along with that of Ge substrate and bare GaAs NWs. (d) Room temperature PL spectra of bare GaAs NWs and GaAs/Ge NWs with different growth temperatures. 


\section{REFERENCES}

(1) Appenzeller, J.; Knoch, J.; Bjork, M. T.; Riel, H.; Schmid, H.; Riess, W. Toward Nanowire Electronics. IEEE Trans. Electron Devices 2008, 55 (11), 2827-2845.

(2) Yan, R.; Gargas, D.; Yang, P. Nanowire Photonics. Nat. Photonics 2009, 3 (10), 569 576.

(3) Claudon, J.; Bleuse, J.; Malik, N. S.; Bazin, M.; Jaffrennou, P.; Gregersen, N.; Sauvan, C.; Lalanne, P.; Gérard, J.-M. A Highly Efficient Single-Photon Source Based on a Quantum Dot in a Photonic Nanowire. Nat. Photonics 2010, 4 (3), 174-177.

(4) Liu, X.; Long, Y.-Z.; Liao, L.; Duan, X.; Fan, Z. Large-Scale Integration of Semiconductor Nanowires for High-Performance Flexible Electronics. ACS Nano 2012, $6(3), 1888-1900$.

(5) Wallentin, J.; Anttu, N.; Asoli, D.; Huffman, M.; Aberg, I.; Magnusson, M. H.; Siefer, G.; Fuss-Kailuweit, P.; Dimroth, F.; Witzigmann, B.; et al. InP Nanowire Array Solar Cells Achieving 13.8\% Efficiency by Exceeding the Ray Optics Limit. Science (80-. ). 2013, 339 (6123), 1057-1060.

(6) Aberg, I.; Vescovi, G.; Asoli, D.; Naseem, U.; Gilboy, J. P.; Sundvall, C.; Dahlgren, A.; Svensson, K. E.; Anttu, N.; Bjork, M. T.; et al. A GaAs Nanowire Array Solar Cell With 15.3\% Efficiency at 1 Sun. IEEE J. Photovoltaics 2016, 6 (1), 185-190.

(7) Tchernycheva, M.; Messanvi, A.; de Luna Bugallo, A.; Jacopin, G.; Lavenus, P.; Rigutti, L.; Zhang, H.; Halioua, Y.; Julien, F. H.; Eymery, J.; et al. Integrated Photonic Platform Based on InGaN/GaN Nanowire Emitters and Detectors. Nano Lett. 2014, 14 (6), 3515-3520.

(8) Peng, K.; Parkinson, P.; Boland, J. L.; Gao, Q.; Wenas, Y. C.; Davies, C. L.; Li, Z.; Fu, L.; Johnston, M. B.; Tan, H. H.; et al. Broadband Phase-Sensitive Single InP Nanowire Photoconductive Terahertz Detectors. Nano Lett. 2016, 16 (8), 4925-4931.

(9) Koester, R.; Sager, D.; Quitsch, W. A.; Pfingsten, O.; Poloczek, A.; Blumenthal, S.; Keller, G.; Prost, W.; Bacher, G.; Tegude, F. J. High-Speed GaN/GaInN Nanowire Array Light-Emitting Diode on Silicon(111). Nano Lett. 2015, 15 (4), 2318-2323.

(10) Sadaf, S. M.; Zhao, S.; Wu, Y.; Ra, Y.-H.; Liu, X.; Vanka, S.; Mi, Z. An AlGaN CoreShell Tunnel Junction Nanowire Light-Emitting Diode Operating in the Ultraviolet-C Band. Nano Lett. 2017, 17 (2), 1212-1218.

(11) Li, K. H.; Liu, X.; Wang, Q.; Zhao, S.; Mi, Z. Ultralow-Threshold Electrically Injected AlGaN Nanowire Ultraviolet Lasers on Si Operating at Low Temperature. Nat. Nanotechnol. 2015, 10 (2), 140-144.

(12) Goldberger, J.; Hochbaum, A. I.; Fan, R.; Yang, P. Silicon Vertically Integrated Nanowire Field Effect Transistors. Nano Lett. 2006, 6 (5), 973-977.

(13) Xiang, J.; Lu, W.; Hu, Y.; Wu, Y.; Yan, H.; Lieber, C. M. Ge/Si Nanowire Heterostructures as High-Performance Field-Effect Transistors. Nature 2006, 441 (7092), 489-493.

(14) Dillen, D. C.; Kim, K.; Liu, E.-S.; Tutuc, E. Radial Modulation Doping in Core-shell Nanowires. Nat. Nanotechnol. 2014, 9 (2), 116-120.

(15) Hwang, Y. J.; Wu, C. H.; Hahn, C.; Jeong, H. E.; Yang, P. Si/InGaN Core/Shell Hierarchical Nanowire Arrays and Their Photoelectrochemical Properties. Nano Lett. 2012, 12 (3), 1678-1682. 
(16) Conesa-Boj, S.; Boioli, F.; Russo-Averchi, E.; Dunand, S.; Heiss, M.; Rüffer, D.; Wyrsch, N.; Ballif, C.; Miglio, L.; Morral, A. F. I. Plastic and Elastic Strain Fields in GaAs/Si Core-Shell Nanowires. Nano Lett. 2014, 14 (4), 1859-1864.

(17) Conesa-Boj, S.; Dunand, S.; Russo-Averchi, E.; Heiss, M.; Ruffer, D.; Wyrsch, N.; Ballif, C.; Fontcuberta i Morral, A. Hybrid Axial and Radial Si-GaAs Heterostructures in Nanowires. Nanoscale 2013, 5 (20), 9633.

(18) Hocevar, M.; Immink, G.; Verheijen, M.; Akopian, N.; Zwiller, V.; Kouwenhoven, L.; Bakkers, E. Growth and Optical Properties of Axial Hybrid III-V/silicon Nanowires. Nat. Commun. 2012, 3, 1266.

(19) Hauge, H. I. T.; Verheijen, M. A.; Conesa-Boj, S.; Etzelstorfer, T.; Watzinger, M.; Kriegner, D.; Zardo, I.; Fasolato, C.; Capitani, F.; Postorino, P.; et al. Hexagonal Silicon Realized. Nano Lett. 2015, 15 (9), 5855-5860.

(20) Madelung, O (ed). Semiconductors: Group IV Elements and III-V Compounds; 1991. Springer-Verlag Berlin Heidelberg, Berlin.

(21) Conesa-Boj, S.; Hauge, H. I. T.; Verheijen, M. A.; Assali, S.; Li, A.; Bakkers, E. P. A. M.; Fontcuberta I Morral, A. Cracking the Si Shell Growth in Hexagonal GaP-Si CoreShell Nanowires. Nano Lett. 2015, 15 (5), 2974-2979.

(22) Migas, D. B.; Borisenko, V. E.; Rusli; Soci, C. Revising Morphology of 〈111〉-Oriented Silicon and Germanium Nanowires. Nano Converg. 2015, 2 (1), 16.

(23) Oehler, F.; Gentile, P.; Baron, T.; Ferret, P.; Den Hertog, M.; Rouviére, J. The Importance of the Radial Growth in the Faceting of Silicon Nanowires. Nano Lett. 2010, 10 (7), 2335-2341.

(24) Jaccodine, R. J. Surface Energy of Germanium and Silicon. J. Electrochem. Soc. 1963, $110(6), 524$.

(25) Jiang, N.; Wong-Leung, J.; Joyce, H. J.; Gao, Q.; Tan, H. H.; Jagadish, C. Understanding the True Shape of Au-Catalyzed GaAs Nanowires. Nano Lett. 2014, 14 (10), 5865-5872.

(26) Goldberger, J.; He, R.; Zhang, Y.; Lee, S.; Yan, H.; Choi, H.-J.; Yang, P. Single-Crystal Gallium Nitride Nanotubes. Nature 2003, 422 (6932), 599-602.

(27) Algra, R. E.; Hocevar, M.; Verheijen, M. A.; Zardo, I.; Immink, G. G. W.; van Enckevort, W. J. P.; Abstreiter, G.; Kouwenhoven, L. P.; Vlieg, E.; Bakkers, E. P. A. M. Crystal Structure Transfer in Core/Shell Nanowires. Nano Lett. 2011, 11 (4), 16901694.

(28) Gorji Ghalamestani, S.; Heurlin, M.; Wernersson, L.-E.; Lehmann, S.; Dick, K. A. Growth of InAs/InP Core-shell Nanowires with Various Pure Crystal Structures. Nanotechnology 2012, 23 (28), 285601.

(29) Yu, X.; Wang, H.; Lu, J.; Zhao, J.; Misuraca, J.; Xiong, P.; von Molnár, S. Evidence for Structural Phase Transitions Induced by the Triple Phase Line Shift in Self-Catalyzed GaAs Nanowires. Nano Lett. 2012, 12 (10), 5436-5442.

(30) Logan, P.; Peng, X. Strain-Modulated Electronic Properties of Ge Nanowires: A FirstPrinciples Study. Phys. Rev. B 2009, 80 (11), 115322.

(31) Cahangirov, S.; Ciraci, S. First-Principles Study of GaAs Nanowires. Phys. Rev. B 2009, 79 (16), 165118.

(32) Karpov, S. V.; Smirnov, M. B.; Novikov, B. V.; Smirnov, A. N.; Shtrom, I. V.; Chirkov, 
E. L.; Tcirlin, G. E.; Bouravleuv, A. D.; Samsonenko, Y. V. Raman Spectra and Structural Peculiarities of GaAs Nanowires. J. Surf. Investig. X-ray, Synchrotron Neutron Tech. 2014, 8 (1), 104-110.

(33) Abstreiter, G. Light Scattering Studies of Semiconductor Heterostructures. J. Vac. Sci. Technol. B Microelectron. Nanom. Struct. 1985, 3 (2), 683.

(34) Attolini, G.; Bosi, M.; Musayeva, N.; Pelosi, C.; Ferrari, C.; Arumainathan, S.; Timò, G. Homo and Hetero Epitaxy of Germanium Using Isobutylgermane. Thin Solid Films 2008, 517 (1), 404-406.

(35) Chang, Y.; Zhang, M.; Deng, C.; Men, C.; Chen, D.; Zhu, L.; Yu, W.; Wei, X.; Di, Z.; Wang, X. Fabrication of High Quality GaAs-on-Insulator via Ion-Cut of Epitaxial GaAs/Ge Heterostructure. Appl. Surf. Sci. 2015, 346, 46-49. 